\title{
Importancia de la competencia argumentativa en el ámbito educativo: una propuesta para su enseñanza a través del role playing online
}

\section{Importance of Argumentative Competence in the Educational Field: A Proposal for its Teaching through Online Role Playing}

\author{
Alba García-Barrera \\ Universidad a Distancia de Madrid. España. \\ alba.garcia@udima.es
}

\begin{abstract}
Resumen
La capacidad argumentativa es esencial en el ámbito educativo, ya que es una competencia que el alumnado necesitará desarrollar para desenvolverse en su vida diaria y transmitir sus pensamientos, defender sus ideas, mantener diálogos abiertos y comprensivos con los demás, etc. Por ello resulta esencial que el profesorado también la adquiera y la sepa poner en marcha en el aula, ayudando a sus estudiantes a adquirir dicha capacidad. Desde la formación universitaria se persigue que los alumnos, como futuros docentes, desarrollen esta competencia a través de las distintas asignaturas a cursar durante la carrera. Sin embargo, en la educación a distancia no resulta tan sencillo poner en práctica esta habilidad, ya que se carece de un entorno físico presencial que favorezca las interacciones síncronas. En este artículo se examinan las ventajas e inconvenientes que presentan algunas de las posibilidades que existen para trabajar la competencia argumentativa en dichos entornos, presentando finalmente el role playing como estrategia de aprendizaje para experimentarla tanto en la formación del profesorado como en el aula.
\end{abstract}

\section{Palabras Clave}

Role playing, argumentación, enseñanza a distancia, aprendizaje experiencial, formación de profesores.

\begin{abstract}
The argumentative capacity is essential in education as it is a competence that students need to develop to get on in their daily life and transmit their thoughts, defend their ideas, maintain open and understanding dialogs with others, etc. It is therefore essential that teachers also gain knowledge about this capacity and implement it in the classroom, helping students acquire this capacity. In Higher Education, it is pursued that students, as future teachers, develop this competence through the various subjects to be taken during the course of studies. However, in distance education, it is not so easy to practice this skill, as there is no physical attendance environment that encourages synchronous interactions. This article discusses the advantages and disadvantages of some of the possibilities for working the argumentative competence in such environments, presenting finally role
\end{abstract}


playing as a learning strategy to experience both in teacher training and in the classroom.

\section{Keywords}

Role playing, argumentation, distance study, experiential learning, teacher education.

\section{Introducción}

En todo proceso de comunicación en el aula se hace necesario que el profesor dialogue con sus alumnos en pos de una metodología activa que logre el aprendizaje significativo de los alumnos. Incentivar su participación, involucrarles y ayudar a que se responsabilicen de su propio aprendizaje se perfila como un continuo interactivo en el que la capacidad argumentativa cobra una especial relevancia. El docente ya no se presenta como mero transmisor de conocimientos, sino que su rol se configura como el de un acompañante cognitivo (Tedesco, 2011) que guía a los estudiantes en su trabajo de aprender a aprender y adquirir aquellas competencias necesarias para la vida. En esta tarea de orientación y ayuda al alumnado, en la cual se convierte en parte central y activa del proceso de enseñanza-aprendizaje, los futuros docentes deben ser capaces de negociar y construir colectivamente significados, contrastar y confrontar opiniones, defender y aportar ideas, convencer, extraer conclusiones, incentivar el debate, favorecer el diálogo abierto y comprensivo, fomentar la reflexión y el razonamiento crítico... Todas ellas habilidades que no sólo deben poseer como futuros maestros, sino que además deben saber cómo desarrollar en su alumnado. Por tanto, la argumentación se posiciona como una de las principales estrategias que han de acontecer en el aula (Gavaldà, Conde, Girondo, Macaya y Viscarro, 2008), y, en consecuencia, debería ser uno de los objetivos a perseguir en la formación del profesorado.

Diversos estudios, como el llevado a cabo por Gavaldà et al. (2008) han demostrado que en general la capacidad argumentativa de los estudiantes universitarios resulta incompleta y poco sólida, tanto por sus contenidos discursivos como por su estructura argumentativa. Esto es debido, en buena medida, a que el profesorado de las distintas etapas educativas apenas introduce en sus clases la enseñanza metódica de la argumentación. Tal y como afirman Camps y Dolz (1995), "no sólo no se enseña a los estudiantes a expresar sus opiniones oralmente o por escrito, a discutir y debatir temas controvertidos, sino que muy pocos manuales de lectura presentan textos argumentativos" (p. 5).

Sin embargo, actualmente vivimos inmersos en una sociedad en la que internet y los medios de comunicación son omnipresentes. Invaden nuestra vida y nuestro quehacer diario, haciendo un uso constante de la persuasión. Desde los anuncios de televisión, los periódicos, las revistas, la publicidad en las calles, la carretera o las apps en nuestro propio móvil, pasando por cualquier noticiero o programa televisivo o radiofónico. Casi desde cualquier lugar y cualquier ámbito se nos transmite un mensaje cuyo objetivo es convencernos de determinada opción: mantener una impresión u opinión concreta sobre una conducta, una idea, un pensamiento, un producto, un personaje... Lo que sucede y aquello que conocemos está sujeto a la información que recibimos, que a su vez condiciona lo que percibimos e incluso lo que pensamos o hacemos. Y la única arma que tenemos al alcance para hacerle frente no es más que nuestro pensamiento crítico, tomando por base la capacidad de argumentación.

Importancia de la competencia argumentativa en el ámbito educativo: una propuesta para su enseñanza a través del role playing online. A. García 
Ambas habilidades, pensamiento crítico y capacidad argumentativa, son complejas y pertenecen al denominado pensamiento de orden superior. Van más allá de mantener y defender una posición o una idea concreta. Consisten en saber diferir de la opinión que nos muestra otra persona, juzgar la información que recibimos y ponerla en entredicho, posicionarse de forma motivada ante un desacuerdo, comprender los diferentes puntos de vista en un conflicto, encontrar y ofrecer alternativas a las razones dadas, cambiar la propia perspectiva o la de los demás... Son tareas que requieren formar parte del ámbito educativo y constituirse como un objetivo didáctico esencial. Además, su utilidad excede con creces lo académico, ya que resultan básicas para una adecuada convivencia en sociedad.

No obstante, aprender a gestionar de forma autónoma este tipo de tareas conlleva una gran complejidad, especialmente en la enseñanza a distancia, donde la falta de sincronía y de presencia física reduce y dificulta las posibilidades de ponerlas en práctica. A fin de ofrecer algunas alternativas en este sentido, en el presente artículo se exponen algunas estrategias didácticas que pueden ayudar a alcanzar dicho objetivo, finalizando con la presentación de una propuesta dirigida a la formación del profesorado y que fue implementada en una universidad a distancia.

\section{Posibilidades didácticas para favorecer la capacidad argumentativa}

Muchas de las propuestas que se pueden realizar en entornos virtuales de aprendizaje parten de una perspectiva docente presencial, tratando de extrapolar prácticas que de forma tradicional han sido llevadas a cabo en las aulas. Otras resultan novedosas y tienen su origen en dichos entornos, adaptándose y partiendo de sus propias características y posibilidades. Por ello, a continuación se exponen los cuatro modelos de instrucción que recoge López Aymes (2012) y que pueden servir como marco para trabajar el pensamiento crítico y la capacidad argumentativa en cualquiera de las estrategias presentadas en el presente apartado.

El primero de ellos es el modelo de evaluación procesual, de Mayer y Goodchild (1990), que se centra en el desarrollo de competencias metacognitivas y autorregulatorias, y más específicamente, a las habilidades de comprensión y evaluación de argumentos por medio de un intento activo y sistemático por llegar a entender y evaluar las ideas o argumentos tanto de los demás como los propios. Se pone énfasis en la comprensión de las aseveraciones planteadas, las evidencias ofrecidas y las explicaciones subyacentes. Además se solicita a los estudiantes que reconozcan y analicen las partes constitutivas de los distintos argumentos. La evaluación de dichas habilidades se realiza por medio de preguntas abiertas en pruebas de tipo ensayo.

El segundo modelo es el de pensamiento dialógico, de Paul et al. (1995). Estos autores sugieren una estrategia para evitar las perspectivas únicas, los prejuicios, la imparcialidad y la irracionalidad. Se invita a que el estudiante asuma otros roles y razone opiniones contrarias a la suya, conociendo en profundidad sus debilidades. Para ello proponen una serie de estrategias: a) afectivas, para practicar la imparcialidad y el pensamiento independiente; b) macrocognitivas, para compartir situaciones análogas, analizar o evaluar argumentos, pensamientos, teorías, etc.; c) microcognitivas, para comparar y contrastar ideales, pensar con precisión, usar vocabulario crítico, etc.

El tercer modelo que recoge esta autora es la comunidad de investigación, de Matthew Lipman (1998), quien apuesta por una pedagogía del juicio 
predominantemente filosófica, que toma por base una comunidad de investigación en la que el diálogo se posiciona como principal herramienta. Sus componentes son: a) la propuesta de un texto, en forma de relato; b) la elaboración del plan de discusión; c) la construcción de la comunidad; d) el uso de ejercicios y actividades para la discusión; y e) el fomento de compromisos para el futuro.

Por su parte, el cuarto y último modelo es el de la controversia, de Beltrán y Pérez (1996), que se basa en el planteamiento de un conflicto académico en el que las ideas, conclusiones y teorías de unos estudiantes son incompatibles con las de otros, debiendo todos ellos llegar a un acuerdo. Sus fases podrían ser las siguientes: a) seleccionar el tema de discusión, estableciendo posiciones antagónicas de interés para los alumnos; b) organizar la clase en pequeños grupos; c) diseñar materiales que recojan claramente las posiciones de cada grupo; y d) disponer y dirigir la controversia hasta alcanzar un consenso. Entre las condiciones que deben darse para que la controversia sea constructiva, dichos autores citan la distribución cooperativa de las actividades, la diversidad de los grupos, la provisión de información relevante, y la enseñanza de habilidades de resolución de conflictos y procedimientos para la argumentación racional.

Estos cuatro modelos pueden servir de orientación para seleccionar una metodología adecuada a la estrategia que se desea emplear para trabajar la capacidad argumentativa, adaptándola a las necesidades y medios de los que se disponga. A continuación se presentan algunas de ellas, enfocándolas a su implementación en la educación a distancia.

\subsection{Reflexiones individuales}

En los entornos virtuales se ha tratado típicamente de desarrollar la capacidad argumentativa desde algunas asignaturas por medio de propuestas basadas en la reflexión del alumnado. Como punto de partida, se expone un asunto o temática concreta, se propone una lectura o la visualización de una película o documental, se recurre a un extracto de un libro, se ofrece un artículo o noticia, se plantea una búsqueda en internet sobre determinada cuestión, etc. A partir de ello el estudiante debe elaborar su propia opinión examinando las distintas alternativas y puntos de vista, exponiendo a continuación, de forma justificada, los motivos o razones en las que se fundamenta su perspectiva.

En ocasiones el profesor agrega a ese punto de partida unas pautas o preguntas a modo de guía que orienten al estudiante en su reflexión, fomentando la controversia inicial. Adicionalmente se puede llegar a solicitar al alumno que refleje su respuesta a dichas preguntas, invitándole a plasmar el razonamiento que le ha conducido a ofrecer determinada respuesta o a mantener cierta postura.

También se puede conducir a la reflexión individual de los estudiantes por medio de otros recursos o herramientas, tales como las webquest, las webgincanas (Novelino, 2010), las cazas del tesoro, o los objetos de aprendizaje. Sobre la aplicación concreta de estos últimos al asunto que nos ocupa puede encontrarse más información en el trabajo de Cruz, Alfaro y Ramírez (2012).

\subsection{Discusión socrática}

Una estrategia didáctica que puede ser llevada a cabo tanto individualmente como en gran grupo es la discusión socrática.

Importancia de la competencia argumentativa en el ámbito educativo: una propuesta para su enseñanza a través del role playing online. A. García 
Este método se compone de dos partes. La primera, que se podría considerar destructiva, y la segunda, en contraposición, que tendría carácter creativo (De la Torre, 2003). Así, y extrapolándolas al aula, en la primera etapa se parte de las ideas y conocimientos previos del estudiante acerca del tema a tratar, haciéndolos surgir a través del cuestionamiento y permitiendo que éste detecte por sí mismo las contradicciones y posibles contrapuntos existentes en sus concepciones. En la segunda etapa se hace uso de la mayéutica, ayudando al alumno a descubrir la verdad o hallar la solución al problema o disyuntiva propuesta. En esta estrategia resulta primordial emplear sistemáticamente la ironía socrática, consistente en aparentar ignorancia sobre el asunto que se está trabajando, a fin de que el alumno alcance por sí mismos sus propias conclusiones por medio del diálogo establecido.

El diálogo socrático se puede configurar, por tanto, como una estrategia de reflexión e indagación grupal que ofrece resultados inmediatos, incluso sin haber concluido, ya que el proceso resulta tan importante como el resultado a obtener (Prawda, 2011).

En la enseñanza a distancia este método puede ser puesto en marcha tanto de forma síncrona como asíncrona, ya sea mediante foros de debate o videoconferencias.

\subsection{Aprendizaje basado en problemas (ABP)}

Otra metodología que puede llevarse a cabo tanto en entornos síncronos como asíncronos para favorecer la capacidad argumentativa es el Aprendizaje Basado en Problemas (ABP). Consiste en plantear un caso práctico que toma como base un conflicto o dilema. Puede tratarse de un problema matemático, una controversia, un rompecabezas, un proyecto, una comparación, una situación contradictoria, etc. El estudiante o grupo de estudiantes debe tratar de resolver dicho problema a través de la búsqueda de respuestas, el planteamiento de hipótesis y preguntas, la investigación, la toma de decisiones... poniendo en marcha así habilidades de pensamiento complejo que influyen en la adquisición de un aprendizaje significativo.

Según Exley y Dennis (2007), las fases en las que se ha de trabajar esta metodología son las siguientes: a) aclarar términos y conceptos; b) definir los problemas; c) analizar los problemas: preguntar, explicar, formular hipótesis, etc.; d) confeccionar una lista sistemática del análisis; e) formular los resultados de aprendizaje esperados; f) aprender centrándose en dichos resultados; y g) resumir y presentar nueva información.

Morales y Landa (2004), por su parte, consideran que el desarrollo de esta estrategia se produce en ocho fases, dejando la definición del problema y el planteamiento de preguntas para las fases finales. No obstante, y a fin de seguir el modelo constructivista, parece más adecuado partir de la presentación del problema para que sean los propios estudiantes quienes decidan qué datos resultan necesarios para su posterior discusión, análisis y resolución, basándose así en sus esquemas previos de conocimiento. De esta forma se logra que el problema a resolver cobre un verdadero significado, promoviendo así una disposición afectiva positiva y motivando a los estudiantes (García y Occelli, 2012).

Por tanto, mediante esta estrategia se enseña a los estudiantes a argumentar y pensar de forma activa. Establecen conexiones entre conceptos y construyen sus propias representaciones mentales, de modo que "el aprendizaje se vuelve más desafiante, interesante y motivador y puede desembocar en una comprensión y una retención mejoradas" (Zohar, 2006, p.158). 


\subsection{Foros de debate}

Una forma clásica de confrontar opiniones y de fomentar el debate colectivo en los entornos virtuales de enseñanza es mediante el uso de foros. El profesor normalmente elige un tema, que puede formar parte de los contenidos de la asignatura, de un texto de referencia, de un vídeo, una noticia, etc., y los estudiantes reflejan por escrito lo que piensan acerca de él. En ocasiones son los propios alumnos quienes eligen un tema, ya sea en base a sus propios intereses o en relación a una serie de temáticas dadas por el profesor.

En cuanto al rol del profesor en estos foros, suele ser el de moderador, ya que cuando se trata de foros de debate abiertos en los cuales los estudiantes participan de forma voluntaria y sin la intervención del profesor, la discusión normalmente fracasa (Gunawardena, Plass y Salisbury, 2001). Por ello, normalmente el docente hace de mediador y solicita una mayor argumentación o justificación de las ideas expuestas, perfila ideas nuevas, ofrece sugerencias sobre determinadas aportaciones, plantea preguntas para fomentar la reflexión, dirige el debate hacia líneas de mayor interés o polémica, etc. De este modo se incentiva la controversia, de modo que se produzca una mayor diversidad de perspectivas y se conduzca a que los alumnos expresen su opinión y defiendan su punto de vista. Sin embargo, suele suceder que los estudiantes no leen las aportaciones de sus compañeros y se limitan a dar la propia, por lo que se repiten argumentos y perspectivas y no se consigue fomentar un debate verdaderamente enriquecedor. Con suerte algún alumno repasa lo que han dicho sus compañeros y prepara con detenimiento su contribución, a la que posteriormente se une cierto número de compañeros que avalan lo que en ella se expone, resumiendo par de aspectos importantes y afirmando coincidir en el resto. Únicamente se reinterpretan las opiniones ya vertidas, expresándolas con otras palabras, y rara vez se refutan mediante algún argumento de base más bien pobre. La ventaja de este tipo de actividad es que no requiere necesariamente sincronía, ya que cada estudiante puede efectuar su contribución cuando mejor le convenga y leer las que sus compañeros han realizado en diferentes momentos. Esto sumado a la ubicuidad que proporcionan los entornos virtuales favorece la comunicación constante con personas que están lejos, sin importar las posibles limitaciones temporales. Además, al dilatar en el tiempo los ciclos de interacción, se fomenta la reflexión y la madurez de los mensajes que producen los estudiantes (Arango, 2004).

Este tipo de foros, por tanto, favorecería lo que se conoce como diálogos pragmáticos, en línea con el propósito de enseñar, practicar y desarrollar la capacidad argumentativa. Se considera que en el diálogo pragmático el conocimiento se consolida partiendo de la identificación de determinados conceptos y contenidos que son discutidos hasta la formulación de nuevos argumentos, apoyándose en la indagación y en las denominadas filigranas mentales (estrategias de pensamiento crítico utilizadas en las discusiones virtuales) (Arango, 2004).

No obstante, una gran carencia que presentan los foros de debate en cuanto a la implementación de la capacidad argumentativa cuando se trabajan de forma asíncrona, es que se alejan bastante de la realidad del aula, impidiendo al docente poner en marcha tanto sus habilidades lingüísticas orales como determinadas competencias propias de la argumentación, especialmente aquellas que se manifiestan con carácter espontáneo.

Importancia de la competencia argumentativa en el ámbito educativo: una propuesta para su enseñanza a través del role playing online. A. García 


\subsection{Chat y herramientas VoIP}

El chat y las videoconferencias favorecen aquellas habilidades lingüísticas de carácter oral y espontáneo que a nivel argumentativo se han identificado como punto de flaqueza en la anterior propuesta. Sin embargo, esta alternativa carece de aquellas otras fortalezas que presentaban los foros de debate asíncronos, tales como mayor tiempo para reflexionar y poner por escrito la opinión, la capacidad para interpretar y comprender otros puntos de vista, la posibilidad de mantener y establecer contacto con los compañeros sin necesidad de coincidir en horarios, etc.

A través del chat o de herramientas VoIP, que permiten la comunicación de texto, voz y vídeo entre usuarios utilizando como soporte internet, se puede fomentar la capacidad argumentativa oral de una forma muy similar a como se llevaría a cabo en persona, en un aula física. Algunos programas y aplicaciones multiplataforma gratuitas que pueden usarse en este sentido y que facilitan las videoconferencias grupales son: Skype, Hangouts, ChatOn, GroupMe, Fring, MeetingBurner, WebEx, TinyChat, LiveStream, Join.me, FriendCaller, etc.

\subsection{Networking y seminarios interactivos}

Algunas herramientas que posibilitan las videoconferencias grupales ofrecen la posibilidad de realizar pequeños grupos de trabajo dentro de una misma sesión, creando pequeñas salas de conversación independientes. El anfitrión de la sesión, en este caso el profesor, puede realizar una introducción conjunta en gran grupo con los estudiantes, en la cual exponga una situación o problemática concreta, presente un caso o conflicto, etc., y luego solicite a los estudiantes que reflexionen sobre ello, dividiéndolos en pequeños grupos para que debatan e intercambien impresiones y puntos de vista sobre el asunto a tratar. Tras unos minutos se anuncia el cierre de dichas salas de trabajo y se vuelve a la sala principal, en la que el profesor retoma el hilo de la sesión y solicita a los distintos grupos que expongan sus ideas. Puede exigirse que lleguen a unas conclusiones o puntos de encuentro entre las distintas opiniones que se hayan vertido en los pequeños grupos, y que en cada uno de ellos se seleccione un portavoz para que las exponga ante el resto de la clase, por turnos. Una vez expresadas dichas conclusiones por los diferentes portavoces, se puede proceder a la realización de nuevos cuestionamientos para que vuelvan a repensarse y discutirse en pequeños grupos.

Pueden mantenerse los mismos miembros en cada uno, de modo que una vez escuchadas las nuevas aportaciones puedan surgir reinterpretaciones de dichas realidades y se trabaje sobre la comprensión de las perspectivas suscitadas por los otros grupos; o bien pueden efectuarse nuevos grupos, intercambiando los distintos miembros de los equipos, de modo que puedan intercambiar las ideas expuestas en sus respectivos grupos $\mathrm{y}$ aquellas que han sido mencionadas por los portavoces, tomando en consideración los nuevos interrogantes que, así mismo, hayan sido planteados por el profesor.

Este tipo de dinámicas interactivas resultan altamente enriquecedoras, pero siguen careciendo del tiempo adicional que ofrecen los foros de debate, exigiendo además que todo el grupo se conecte al mismo tiempo.

Por tanto, y una vez examinadas las ventajas y debilidades que presentan algunas de las estrategias que pueden emplearse en los entornos virtuales de aprendizaje para trabajar la capacidad argumentativa, se ofrece a continuación una propuesta centrada en la formación del profesorado.

Importancia de la competencia argumentativa en el ámbito educativo: una propuesta para su enseñanza a través del role playing online. A. García 


\section{Propuesta para la formación del profesorado}

La presente propuesta parte de una experiencia implementada en una universidad a distancia, cuyo principal objetivo consistía, principalmente, en lograr que los estudiantes de Magisterio, en tanto futuros docentes, desarrollasen su capacidad argumentativa a la par que reflexionaban sobre cómo integrar y poner en marcha dicha competencia en el aula. Para ello, se hizo uso del role playing como estrategia básica para el aprendizaje.

\subsection{Características del role playing}

Se puede decir que el origen del role playing a nivel pedagógico tuvo lugar en la década de los 60 del siglo pasado, momento en el que Moreno (1959; en Martínez Riera, 2009) introdujo el "psicodrama didáctico o pedagógico", cuyo objetivo consistía en estudiar la capacidad de un individuo para representar diversos papeles por medio de la interpretación.

El juego de roles puede ser individual o múltiple. La diferencia consiste en que en el role playing individual la actividad es llevada a cabo por un único grupo, mientras el resto de participantes actúan como observadores; y en el role playing múltiple son varios grupos quienes realizan un mismo problema a la vez. El primero ofrece la ventaja de que los observadores pueden prestar una mayor atención a la representación, evaluando con detenimiento todo lo que se dice y hace. El segundo, por su parte, hace posible la ulterior comparación del proceso seguido por cada grupo, así como el resultado obtenido (Domene y Romero, 2001).

Según su grado de planificación, las escenificaciones, juegos de roles o role playing, pueden clasificarse como: a) estructuradas, si el discurso y la escena están muy definidas; b) semiestructuradas, si se asigna un guión breve sobre la escena y se reparten unos papeles breves; c) no estructuradas, si únicamente se asignan los papeles; y d) mixtas, si se mezclan dos de dichas variedades (de la Herrán, 2009).

En cuanto a las fases para implementar el role playing, algunos autores mencionan tres: a) preparación, b) representación o realización de la simulación en tiempo real, y c) reflexión o elaboración de conclusiones (de la Herrán, 2009; Jordán y Peñas, 2012). Otros autores, como Martín (1992) o Alonso (2004), establecen una fase previa a la preparación o motivación, en la que se muestra el interés de la escena y se estimula al alumno para que se implique y participe activamente en la representación. Así, quedan definidas cuatro fases: a) entrenamiento y motivación; b) preparación de la dramatización; c) dramatización; y d) comentario o debate.

Centrándonos en el ámbito que nos ocupa, resulta necesario señalar que el roleplaying pedagógico se basa en "perfeccionar, modificar, ampliar un rol, e incluso, crearlo como algo nuevo. [...] Es decir, ayudando a crear en el estudiante una mayor espontaneidad que le permite usar sus propios recursos, con el fin de crear él mismo el rol que mejor se adapte a las demandas del contexto en el que se sitúa" (Martínez Riera, 2009, p. 235).

De esta forma, se puede afirmar que la base del role playing se establece en la dramatización y la improvisación. La dramatización consiste en la representación de una acción efectuada por ciertos personajes en un espacio concreto, y la improvisación en la simulación de algo imprevisto e inventado de forma espontánea desde un estímulo dado

Importancia de la competencia argumentativa en el ámbito educativo: una propuesta para su enseñanza a través del role playing online. A. García 
(Barroso y Fontecha, 1999). La primera puede ayudar a los estudiantes a ponerse en el lugar del otro, comprender perspectivas, opiniones $\mathrm{y}$ formas de ser y actuar, favoreciendo la empatía, mejorando el conocimiento propio y el de los demás, facilitando la adquisición de habilidades socioemocionales, inter e intrapersonales, y fomentando la inteligencia emocional. A través de ambas, especialmente de la improvisación, se incentiva la creatividad y la imaginación de los estudiantes, así como la capacidad de ofrecer respuestas divergentes y rápidas a todo tipo de problemáticas, con el beneficio que ello conlleva para el desarrollo de la capacidad argumentativa.

\subsection{Ventajas del role playing}

Como ya se ha ido mencionando, el role playing presenta múltiples ventajas tanto para la formación del profesorado como para el proceso de enseñanza-aprendizaje, tales como la motivación, la empatía y la perspectiva social (Martín, 1992); despiertan interés, activan la participación espontánea de los espectadores, mantienen la expectativa del grupo; enseña a aprender a aprender, a aprender haciendo, fomenta la creatividad y el aprendizaje por descubrimiento, invita a la reflexión y a la interacción grupal, favorece la resolución de problemas, posibilita la formulación de ideas y la expresión de sentimientos, etc. (Pérez Gutiérrez, 2004).

Otra de las ventajas que presenta el role playing es que puede llegar a usarse como técnica de evaluación, ya que "facilita al docente la observación y comprobación de los aprendizajes alcanzados por los estudiantes mediante una actividad lúdica, en donde ellos deben aplicar los conceptos y elementos estudiados" (Gaete-Quezada, 2011, p. 304).

Además, implica la adquisición de "competencias técnicas más específicas, como, por ejemplo, el trabajo en equipo, el liderazgo, la comunicación, la responsabilidad y la tolerancia, lo que ofrece la oportunidad de alcanzar un aprendizaje significativo más real" (Gaete-Quezada, 2011, p. 304).

No obstante, algunas de las competencias que pueden destacarse a la hora de favorecer y poner en marcha el desarrollo de la capacidad argumentativa son el pensamiento crítico, el aprendizaje significativo, el aprendizaje cooperativo y el aprendizaje dialógico.

\subsubsection{Pensamiento crítico}

Paul y Elder (2006) proponen un modelo para alcanzar y desarrollar el pensamiento crítico. Afirman que los pensadores críticos aplican de forma cotidiana los estándares intelectuales a los elementos del razonamiento para desarrollar rasgos intelectuales. Consideran como estándares intelectuales la claridad, la precisión, la profundidad, la amplitud y el significado. Definen como elementos del pensamiento los siguientes: a) el propio punto de vista, que puede servir como marco de referencia, perspectiva $u$ orientación; b) el propósito, meta u objetivo a conseguir; c) la cuestión, problema o asunto a tratar; d) la información, datos, hechos, observaciones y/o experiencias de las que se dispone; e) las interpretaciones, inferencias, soluciones y/o conclusiones que se pueden alcanzar; f) los conceptos, teorías, definiciones, axiomas, leyes, principios o modelos que pueden servirnos de guía; g) los supuestos, presuposiciones y prejuicios que generamos; y h) las consecuencias e implicaciones que se pueden derivar de todo ello. Y, por último sitúan como virtudes intelectuales la integridad intelectual, la confianza en la razón, la autonomía intelectual, la humildad intelectual, el coraje intelectual, la perseverancia intelectual, la empatía intelectual y la justicia de

Importancia de la competencia argumentativa en el ámbito educativo: una propuesta para su enseñanza a través del role playing online. A. García 
pensamiento.

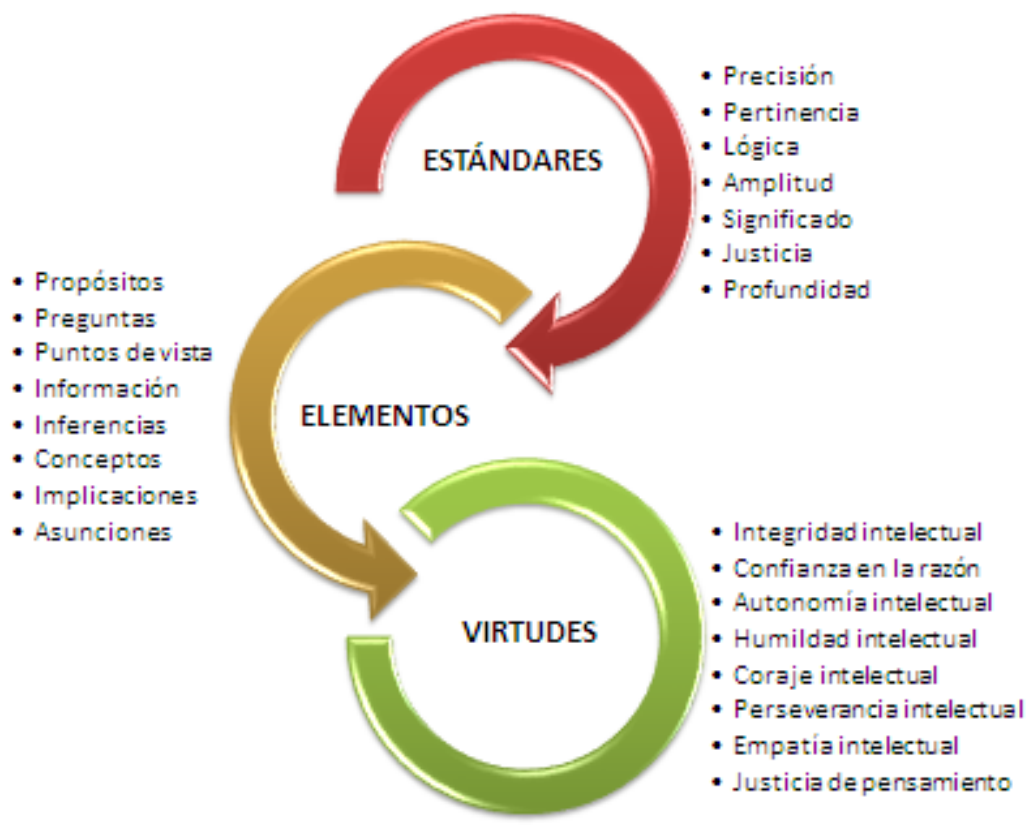

Figura 1. La práctica del pensamiento crítico (Paul y Elder, 2006).

Así, tal y como muestra la Figura 1, Paul y Elder (2006) indican que los estándares deben ser aplicados a los elementos mientras se aprende a desarrollar las virtudes intelectuales.

\subsubsection{Aprendizaje significativo}

Bajo el prisma del constructivismo, el discente se perfila como activo procesador de información, pero a su vez como buscador de información. No se limita a asimilar estímulos que desencadenen cierta respuesta, sino que busca, selecciona y extrae información de su entorno, interpretándola según sus propias expectativas, experiencias y/o conocimientos previos, y atribuyéndole un significado en consecuencia (Rivas Navarro, 2008). Anclar los nuevos conocimientos, competencias e ideas a los esquemas que ya se poseen es la base del aprendizaje significativo, que junto al aprendizaje experiencial permite al estudiante recordar mejor y más fácilmente aquello que se le desea enseñar, yendo más allá de la simple memorización o repetición mecánica de contenidos.

El role playing fomenta el aprendizaje significativo al ofrecer una experiencia en primera persona a los estudiantes y poner en marcha toda una serie de competencias implícitas, como las que ya han sido mencionadas al comienzo de este apartado.

\subsubsection{Aprendizaje cooperativo}

Tanto para los futuros maestros como para casi cualquier profesión, la colaboración con otras personas se presenta como una necesidad diaria. El intercambio de propuestas, opiniones, sugerencias y/o críticas suele darse con frecuencia en el entorno laboral, cuanto más en el aula entre los estudiantes. Por ello, se hace necesaria la adquisición de habilidades interpersonales y el desarrollo de la capacidad argumentativa, a fin de aprender a dialogar y debatir, a cobrar perspectiva sobre la cuestión que se aborda, empatía sobre la opinión vertida por los demás, preparación para fundamentar adecuadamente las propias ideas, comprender las razones de los otros y rebatirlas en su

Importancia de la competencia argumentativa en el ámbito educativo: una propuesta para su enseñanza a través del role playing online. A. García 
caso, etc. García y Occelli (2012) consideran que bajo esta modalidad se generan las condiciones imprescindibles para lograr un aprendizaje social como resultado de la interacción comunicativa.

Por otra parte, el aprendizaje cooperativo puede resultar altamente significativo cuando ocurre entre personas con perspectivas opuestas y diferentes representaciones (De Vries, Lund y Baker, 2002). Esto es debido a que el aprendizaje puede considerarse un proceso social mediado por el lenguaje, que avanza mediante la interpretación, la negociación y la construcción conjunta de significados (García y Occelli, 2012). De esta forma, y concretamente en los entornos virtuales de aprendizaje, se considera que el objetivo de los debates y discusiones consiste en que los estudiantes exterioricen, articulen y negocien diversas alternativas, produciendo la reflexión acerca del significado que poseen los argumentos presentados y ampliando la comprensión de los mismos (Monsalve, 2012).

Por todo ello, la argumentación se perfila como un importante mecanismo de cara a la producción de debates provechosos y la elaboración de actividades constructivas en el ámbito educativo (Dillenbourg y Schneider, 1995; Baker, 1999; Savery y Duffy, 1996; en Monsalve, 2012).

\subsubsection{Aprendizaje dialógico}

El aprendizaje dialógico se centra en la dimensión intrapsicológica e intersubjetiva del aprendizaje, reclamando que los procesos de enseñanza-aprendizaje se estudien desde una concepción comunicativa que tenga en cuenta el contexto en el que se producen las interacciones sociales, prestando una mayor atención y fomentando éstas (Aubert, García y Racionero, 2009). Para ello, se han tomado como referente las teorías y aportaciones de autores como Freire (1970), Habermas (1987), Mead (1973), Vygotsky (1996), Rogoff (1993), Wells (2001), Wenger (1988, 1991), Scribner (1984, 1988) o Bruner (1996) (en Aubert, García y Racionero, 2009).

Las interacciones que se favorecen por medio del aprendizaje dialógico pueden fomentar, entre otros aspectos, el aprendizaje instrumental y la creación de sentido personal y social, ya que se encuentran orientadas a principios solidarios en los que la igualdad y la diferencia son valores compatibles y que se enriquecen mutuamente (Aubert, Flecha, García, Flecha y Racionero, 2008). Además, el aprendizaje dialógico se centra en el diálogo igualitario, que a su vez se sustenta en la acción comunicativa, abriendo un diálogo entre todas las personas implicadas, situadas en un plano de igualdad y tomando como base argumentos de validez y no de poder, generando así entendimiento y aprendizaje (Duque, de Mello y Gabassa, 2009). Por tanto, sus principios suponen una gran ayuda para la adquisición de la competencia argumentativa y se pueden resumir de la siguiente forma (Figura 2):

Importancia de la competencia argumentativa en el ámbito educativo: una propuesta para su enseñanza a través del role playing online. A. García 


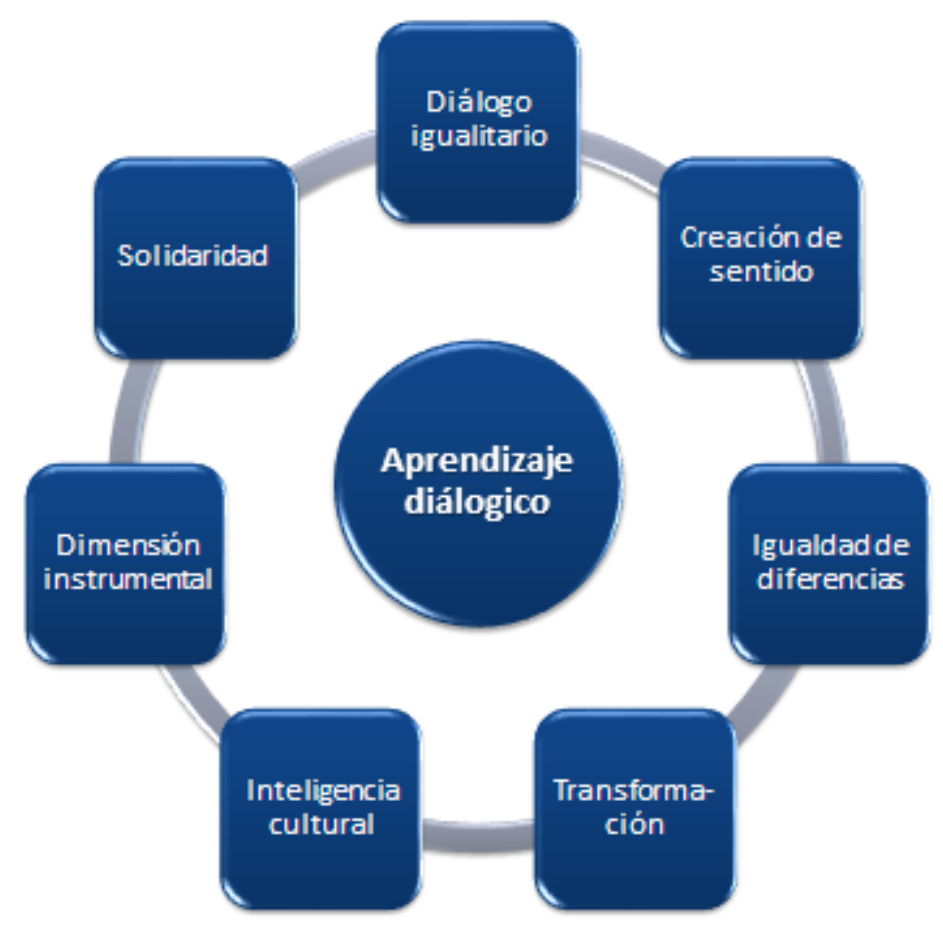

Figura 2. Aprendizaje dialógico.

- Diálogo igualitario, en el cual las aportaciones se valoran según la validez de los argumentos aportados y no en función de las relaciones de poder o jerarquías sociales establecidas.

- Inteligencia cultural, que incluye las inteligencias académica, práctica y comunicativa, mediante las cuales se pueden establecer acuerdos a través del lenguaje.

- Transformación, que hace plausibles los cambios en las propias personas y su contexto.

- Dimensión instrumental, que considera todos los conocimientos imprescindibles para vivir en la sociedad actual.

- Creación de sentido, que toma como base un aprendizaje que parte de la interacción y de las necesidades de las propias personas, quienes guían su propio proceso de aprendizaje.

- Solidaridad, como pieza clave en toda práctica educativa democrática que pretenda superar el fracaso escolar y la exclusión social.

- Igualdad de diferencias, donde la diversidad se valore en tanto elemento positivo facilitador de riqueza cultural.

La implementación de dichos principios puede sentar la base para trabajar y lograr un role playing de calidad, por lo que deben ser tomados en consideración no sólo para alcanzar y desarrollar la capacidad argumentativa del alumnado, sino para cuidar el proceso por el cual se adquiera. 


\subsection{El role playing interactivo}

Las actividades instructivas de discusión online se han impuesto en los últimos años, centrándose actualmente la investigación en la puesta en marcha de estrategias innovadoras que permitan a los estudiantes adquirir un conocimiento más profundo y una mayor interacción en los entornos virtuales de aprendizaje (Hou, 2012). A fin de alcanzar estos objetivos, se propone como modelo de trabajo en el aula virtual el role playing interactivo.

En función de la temática que se trabaje, el juego de roles puede tener diversas modalidades. El role playing que se plantea dentro de la presente propuesta se puede encuadrar dentro de lo que De la Herrán (2009) denomina como "juicio". Consiste en proponer un tema o asunto polémico sobre el que se pueden ejecutar diferentes roles: a) un equipo defensor, que incluye un portavoz a modo de abogado; b) un equipo fiscal, encargado de demostrar la "culpabilidad" del asunto juzgado; c) un juez, que coordinará todo el proceso; d) un equipo de secretarios, que tomarán notas para redactar el acta del juicio; e) el guardia jurado, encargado de presentar el acto; f) el jurado propiamente dicho, que decidirá de forma democrática en base a lo escuchado si el tema juzgado es culpable o inocente; y g) el público de la sala.

Como ya se ha visto, en función del tipo de role playing (estructurado, semiestructurado o no estructurado), los distintos equipos podrán preparar previamente los argumentos que van a utilizar durante el juicio, de modo que se puede trabajar la capacidad argumentativa desde diversas perspectivas: fomentando la espontaneidad de las respuestas, incentivando la solidez de los argumentos, favoreciendo el pensamiento crítico, promoviendo una evaluación pausada de los pros y contras, impulsando la empatía y la capacidad de persuasión, etc.

\subsection{Características de la experiencia}

La propuesta que se realiza en el presente artículo parte de una experiencia puesta en marcha en el Grado de Magisterio de Educación Primaria de la Universidad a Distancia de Madrid, concretamente dentro de la asignatura "Observación e innovación en el aula". En dicha experiencia se pretendía desarrollar las capacidades propias de la argumentación tanto a nivel escrito como oral, constituyéndose para ello dos fases: la confección de un documento escrito, en el que se plasmaba la estructura del role playing y su posible aplicación dentro del aula, y la representación del role playing.

En la primera fase se apostó por un role playing semiestructurado, en el que los estudiantes ponían por escrito los distintos roles y explicaban brevemente el perfil y posición que adquiría cada uno de ellos durante el juicio. Esta puesta en común, referente al diseño del juicio y de su aplicación al aula, se efectuó por medio de la herramienta Google Docs, ya que, como se ha dicho, se trataba de estudiantes de Magisterio de una universidad a distancia. Dicha herramienta hace posible la comunicación intragrupo de forma síncrona, a través del chat, o asíncrona, por medio de mensajes electrónicos que se envían por vía interna y que llegan al correo personal de cada participante.

En la segunda fase, relativa a la escenificación del role playing, en la que cada estudiante debía interpretar los roles que tuviera asignados dentro del juicio previamente diseñado, se hizo uso de la herramienta Skype, que permite realizar llamadas y videoconferencias. Además ofrece la posibilidad de integrar aplicaciones adicionales, como CallNote y VoiceMaster, motivo por el cual se optó por el uso de dicha

Importancia de la competencia argumentativa en el ámbito educativo: una propuesta para su enseñanza a través del role playing online. A. García 
herramienta en lugar de otras posibilidades.

A continuación se describirá la experiencia desarrollada en mayor detalle.

\subsection{Estructura de la propuesta}

\section{Fase 1: confección del documento escrito.}

Dentro de esta experiencia lo primero que hicieron los estudiantes fue unirse a un grupo. Para ello, en el aula virtual de la asignatura, en la plataforma Moodle, justo debajo de la actividad y con el objeto de facilitar la formación de los distintos grupos, se habilitó una consulta en la que cada estudiante debía adscribirse a un grupo según su disponibilidad (mañana, tarde o noche; de cara a la posterior realización síncrona del trabajo). De forma paralela, se abrió un foro para la comunicación asíncrona entre los integrantes de cada grupo, a fin de que pudieran intercambiar sus usuarios de Skype para realizar el trabajo y pensar en qué consistiría su role playing.

Una vez hechos los equipos y para comenzar a crear el trabajo junto con el resto de integrantes, se hizo uso de la plataforma Google Docs.

En cuanto a los contenidos del trabajo escrito, se debía tener en cuenta que se trataba de futuros profesores, por lo que era importante que reflexionasen sobre cómo integrarían este tipo de dinámicas en sus clases. Por tanto, el trabajo a realizar debía incorporar los siguientes elementos:

- Una portada que incluyese: a) el título o temática del role playing; y b) los autores que ejecutarían los distintos roles (nombre y apellidos de la persona encargada de interpretar cada papel en el diálogo). Por ejemplo: "Natalia López. Roles: Fulanito y Rogelio", "Ramón Gómez: Fiorentina y Ofelio", etc.

- Una breve descripción del juicio que se pondría en marcha (aproximadamente 10 líneas), prestando especial atención a los argumentos aportados por cada rol.

- Un listado con los roles y las principales características de cada papel en el diálogo. Por ejemplo: "Fulanito Pérez: Acusado. Tímido y sereno. Se le acusa de...", "Rogelio Vargas: Juez. Serio y altivo. Será imparcial ante los hechos...", "Fiorentina Vargas: Abogada del acusado. Dicharachera y vivaz. Defenderá los hechos de forma implacable...", etc.

- Cómo se utilizaría en clase (descripción de la implementación en el aula).

- Enlace de Dropbox a la grabación del role playing.

\section{Fase 2: representación del role playing.}

Para llevar a cabo la representación del role playing que se había diseñado en la parte escrita, se debía concertar un breve encuentro con los compañeros del grupo. Para ello, se hizo uso del foro habilitado en el aula virtual, en el que uno de los miembros facilitaba un Doodle para establecer la disponibilidad de cada participante.

Antes del encuentro, uno de los miembros del equipo debía descargar e instalar en su equipo el programa CallNote, a fin de realizar la grabación del juicio. Además, se puso a disposición de los estudiantes una aplicación adicional, por si deseaban crear distintas

Importancia de la competencia argumentativa en el ámbito educativo: una propuesta para su enseñanza a través del role playing online. A. García 
voces para los personajes: la aplicación VoiceMaster, que permite dar diferentes tonos a la voz de la persona mientras habla. En el aula virtual se ofrecieron varias guías de ayuda y videotutoriales sobre cómo llevar a cabo dicho proceso.

Una vez concertado el encuentro, los estudiantes debían usar Skype, activar CallNote y, en su caso, VoiceMaster, y proceder a la representación del role playing. Cuando ésta terminase, se debía incluir en el trabajo escrito el enlace de Dropbox que generase el programa, a fin de poder valorarlo para su calificación en la asignatura.

\section{Conclusiones}

Esta experiencia permitió a los estudiantes desarrollar una serie de habilidades interpersonales y cognitivas de orden superior, además de fomentar la interacción en el aula virtual, respondiendo así a dos de las cuestiones que los últimos estudios de investigación acerca de las estrategias de discusión online buscan resolver (Hou, 2012).

Así, a través de la experiencia realizada se logró fomentar el aprendizaje significativo y cooperativo, la capacidad de argumentación, la puesta en común de ideas, el debate, las habilidades interpersonales, etc., ya que debían consensuar con sus compañeros de grupo las características del juicio que deseaban efectuar, los distintos roles que estarían presentes y aquellos que se encargarían de ejecutar durante la representación final.

Para implementar la representación del juicio se pueden utilizar diversos programas gratuitos que permiten la interacción síncrona entre los participantes, y que además proporcionan la posibilidad de grabar la sesión para su posterior visualización y consulta. Así, por ejemplo, en lugar de la combinación elegida de Skype con CallNote y VoiceMaster, se podría haber utilizado Google Hangouts, incorporando la opción Hangout on the air, que cuelga el vídeo directamente en YouTube. De ambas formas se puede compartir ulteriormente la grabación de la sesión para que la pueda evaluar el propio profesor o bien otros compañeros. De este modo, los distintos participantes pueden analizar sus propios comportamientos y los de sus compañeros, ayudándoles a adquirir, desarrollar y/o inhibir determinadas conductas, habilidades, competencias o conocimientos (Domene y Romero, 2001). Por tanto, una opción interesante consistiría en incluir en esta propuesta una autoevaluación seguida de una evaluación por pares.

Los resultados de esta experiencia resultaron en general positivos, obteniéndose una alta participación de los estudiantes en la actividad, que fue elegida libremente por la mayoría de los matriculados. Además, según la opinión de los estudiantes implicados, el trabajo supuso una experiencia atractiva, motivadora y enriquecedora para su formación como futuros docentes. Como único punto negativo algunos alumnos hicieron alusión a la necesidad de compatibilizar horarios que les permitiesen interpretar y grabar el role playing de forma síncrona con sus compañeros dentro de las franjas establecidas.

En general, se podría afirmar que una de las mayores ventajas obtenidas a través de la experiencia que se plantea en este artículo como propuesta fue el fomento del aprendizaje cooperativo, metodología que a menudo se asocia a etapas no universitarias pero que se ajusta especialmente al actual diseño de los estudios superiores, "donde la adquisición de competencias a través del trabajo práctico se torna una parte central de la formación del alumnado" (Olmos y Martínez, 2014, p. 4).

Además, en los actuales planes de estudio es necesario que el profesorado universitario comprenda que las clases magistrales deben permanecer en un plano

Importancia de la competencia argumentativa en el ámbito educativo: una propuesta para su enseñanza a través del role playing online. A. García 
secundario, ya que su rol ya no consiste en la mera transmisión de conocimientos, sino que debe ayudar a que sean los propios estudiantes quienes los generen, pasando a ser ellos los principales protagonistas de su proceso de aprendizaje (Pérez-Pérez, del Moral y Povedano, 2014). Así, en esta experiencia se permitió que los futuros maestros de Primaria tomasen el control de su propio ritmo y proceso de aprendizaje en la actividad, diseñando con sus iguales el tema, los roles y el guión a interpretar.

Experimentar en primera persona el juego de roles permitió a los futuros maestros comprender las ventajas que esta estrategia posee para los niños, ya que supone una actividad determinada en gran medida por su propia iniciativa e interés, permitiéndoles elegir su contenido, participantes y medios necesarios para su ejecución (Rodríguez, Govea y Serra, 2013).

Esta experiencia también permitió llevar la oralidad al aula virtual, más escasa en estos entornos que en los presenciales y fundamental para desarrollar y favorecer el diálogo entre los participantes (España, Rueda y Blanco, 2013), que se comunicaron de forma activa y constante tanto durante la preparación del role playing como en la escenificación del mismo.

Esta actividad también fomentó en los futuros maestros "una forma de afrontar los problemas basada en el pensamiento crítico, potenciando a su vez el diálogo y el trabajo colaborativo" (Abella y Grande, 2010; citados en España, Rueda y Blanco, 2013, p. 765), tan necesarios para su profesión.

Por todo ello, se puede concluir que la propuesta presentada constituye una buena oportunidad tanto para el aprendizaje de los futuros maestros, como para el enriquecimiento de la formación en los entornos virtuales de aprendizaje.

En resumen, la introducción del role playing en el aula mejora la experiencia educativa, ya que favorece "el pensamiento crítico y las habilidades analíticas, ofreciendo a los estudiantes un nivel más profundo en la dinámica de intercambio político, el fomento de las competencias de comunicación oral y escrita y el fomento de la confianza de los estudiantes" (Krain y Lantis, 2006, p. 396). En este sentido el juego de roles se sitúa como una buena estrategia para fomentar numerosas habilidades propias de la competencia argumentativa del alumnado. Además, el role playing "completa la formación de la comunicación verbal y no verbal y, por tanto, permite una mejor integración de la persona en los grupos a los que pertenece" (Martínez Riera, 2009, p. 227). De ello se deduce que dicha metodología también resulta idónea para conseguir un clima de aula adecuado y construir una comunidad de aprendizaje (virtual o no) en la que la inclusión cobre un verdadero significado.

Todos estos beneficios, sumados a los ya mencionados en el presente artículo, avalan sólidamente el empleo de estrategias de enseñanza-aprendizaje como las expuestas, en las cuales el estudiante sea protagonista de su aprendizaje e interactúe tanto con sus compañeros como con sus propios conocimientos y habilidades (Gaete-Quezada, 2011). Además, y en base a lo examinado en el apartado 2 (ver Tabla 1), el role playing de tipo juicio se posiciona como uno de los mejores métodos para perfeccionar y desarrollar la capacidad argumentativa del alumnado. 


\begin{tabular}{|c|c|c|c|c|c|c|c|c|}
\hline \multirow{2}{*}{\multicolumn{2}{|c|}{ VENTAIAS }} & \multicolumn{7}{|c|}{ ESTRATEGIAS } \\
\hline & & \multirow{2}{*}{$\begin{array}{c}\begin{array}{c}\text { Reflexiones } \\
\text { individuales }\end{array} \\
\mathrm{x}\end{array}$} & \multirow{2}{*}{\begin{tabular}{|c|}
$\begin{array}{c}\text { Discusión } \\
\text { socrática }\end{array}$ \\
$\mathrm{x}$ \\
\end{tabular}} & \multirow{2}{*}{\begin{tabular}{|c|}
$\begin{array}{c}\text { Aprendizaje } \\
\text { basado en } \\
\text { problemas }\end{array}$ \\
$\mathrm{X}$
\end{tabular}} & \multirow{2}{*}{\begin{tabular}{|c|}
$\begin{array}{c}\text { Foros de } \\
\text { debate }\end{array}$ \\
$\mathrm{X}$ \\
\end{tabular}} & \multirow{2}{*}{\begin{tabular}{|c|}
$\begin{array}{c}\text { Chat } y \\
\text { Volp }\end{array}$ \\
$x$ \\
\end{tabular}} & \multirow{2}{*}{$\begin{array}{c}\text { Networking } \\
y \\
\text { seminarios } \\
\end{array}$} & \multirow{2}{*}{\begin{tabular}{|c} 
Propuesta \\
$\mathrm{x}$
\end{tabular}} \\
\hline Forma de & Individual & & & & & & & \\
\hline trabajo & Grupal & & $x$ & $\mathrm{x}$ & $x$ & & $x$ & $x$ \\
\hline \multirow{2}{*}{ Carácter } & Síncrona & & $x$ & $\mathrm{x}$ & $x$ & $\mathrm{x}$ & $\mathrm{x}$ & $x$ \\
\hline & Asíncrona & $x$ & & $x$ & $x$ & & & $x$ \\
\hline \multirow{2}{*}{ Comunicación } & Oral & & $\mathrm{x}$ & $\mathrm{x}$ & $\mathrm{x}$ & $x$ & $\mathrm{x}$ & $x$ \\
\hline & Escrita & $\mathrm{x}$ & & $\mathrm{x}$ & $x$ & $\mathrm{x}$ & & $\mathrm{x}$ \\
\hline \multirow{4}{*}{ Habilidades } & $\begin{array}{c}\text { Pensamiento } \\
\text { crítico }\end{array}$ & $\mathrm{x}$ & $\mathrm{x}$ & $x$ & $x$ & $\mathrm{x}$ & $\mathrm{x}$ & $\mathrm{x}$ \\
\hline & $\begin{array}{l}\text { Aprendizaje } \\
\text { significativo }\end{array}$ & $x$ & $\mathrm{x}$ & $\mathrm{x}$ & $x$ & $\mathrm{x}$ & $\mathrm{x}$ & $x$ \\
\hline & $\begin{array}{l}\text { Aprendizaje } \\
\text { cooperativo }\end{array}$ & & & $\mathrm{x}$ & & & $\mathrm{x}$ & $\mathrm{x}$ \\
\hline & $\begin{array}{c}\text { Aprendizaje } \\
\text { dialógico }\end{array}$ & & $x$ & & & & & $x$ \\
\hline \multirow{4}{*}{ Modelo } & $\begin{array}{c}\text { Evaluación } \\
\text { procesual }\end{array}$ & $\mathrm{x}$ & $\mathrm{x}$ & $\mathrm{x}$ & $x$ & $\mathrm{x}$ & $\mathrm{x}$ & $\mathrm{x}$ \\
\hline & $\begin{array}{c}\text { Pensamiento } \\
\text { dialógico }\end{array}$ & $x$ & $x$ & $\mathrm{x}$ & $x$ & $\mathrm{x}$ & $\mathrm{x}$ & $x$ \\
\hline & $\begin{array}{l}\text { Comunidad de } \\
\text { investigación }\end{array}$ & & & $x$ & $x$ & & $x$ & $x$ \\
\hline & Controversia & & & $x$ & & & $\mathrm{x}$ & $\mathrm{x}$ \\
\hline
\end{tabular}

Tabla 1. Posibilidades didácticas para el desarrollo de la competencia argumentativa Fin de la redacción del artículo: noviembre de 2014

García-Barrera, A. (2015). Importancia de la competencia argumentativa en el ámbito educativo: una propuesta para su enseñanza a través del role playing online. RED, Revista de Educación a Distancia. 45(4). Consultado el (dd/mm/aaaa) en httb://www.um.es/ead/red/45

\section{Referencias bibliográficas}

Alonso, J.M. (2004). La educación en valores en la institución escolar. Planeaciónprogramación. México, D.F.: Plaza y Valdés.

Arango, M.L. (2004). Foros virtuales como estrategia de aprendizaje. Revista Debates Latinoamericanos, 2(2), 1-13.

Aubert, A., Flecha, A., García, C., Flecha, R. y Racionero, S. (2008). Aprendizaje dialógico en la sociedad de la información. Barcelona: Hipatia.

Aubert, A., García, C. y Racionero, S. (2009). El aprendizaje dialógico. Cultura y Educación, 21(2), 129-139. Recuperado el 15 de octubre de 2014 de http://personal.us.es/aguijim/05_06_Aprendizaje_dialogico.pdf

Barroso, C. y Fontecha, M. (1999). La importancia de las dramatizaciones en el aula de ELE: una propuesta concreta de trabajo en clase. ASELE, 10,107-113. Recuperado el 9 de noviembre de 2014 de http://cvc.cervantes.es/ensenanza/biblioteca_ele/asele/pdf/10/10_0105.pdf 
Camps, A. y Dolz, J. (1995). Enseñar a argumentar: un desafío para la escuela actual. Comunicación, lenguaje y educación, 25, 5-8. Recuperado el 28 de octubre de 2014 de http://dialnet.unirioja.es/descarga/articulo/2941554.pdf

Cruz, A., Alfaro, J. y Ramírez, M. (2012). Objeto de aprendizaje abierto para la formación docente orientado a desarrollar competencias de pensamiento crítico con énfasis en habilidades cognitivas. Revista Iberoamericana de Educación a Distancia, 15(1), 103-125. DOI: http://dx.doi.org/10.5944/ried.1.15.779

De la Herrán, A. (2009). Técnicas de enseñanza basadas en la cooperación. En J. Paredes y A. de la Herrán (Coords.), La práctica de la innovación educativa (pp. 279-307). Madrid: Síntesis.

De la Torre, A. (2003). El método socrático y el modelo de van Hiele. Lecturas Matemáticas, 24, 99-121. Recuperado el 16 de noviembre de 2014 de http://www.scm.org.co/aplicaciones/revista/Articulos/733.pdf

De Vries, E., Lund, K. y Baker, M. (2002). Computer-mediated epistemic dialogue: explanation and argumentation as vehicles for understanding scientific notions. The journal of the learning sciences, 11(1), 63-103. DOI: http://dx.doi.org/10.1207/S15327809JLS1101_3

Domene, S. y Romero, R. (2001). Estrategias didácticas para el desarrollo formativo y profesional. En J.A. Morales y B. Bermejo (Coords), Introducción a la formación y desarrollo de recursos humanos (pp. 31-47). Sevilla: Universidad Internacional de Andalucía.

Duque, E., de Mello, R.R. y Gabassa, V. (2009). Aprendizaje dialógico. Base teórica de las comunidades de aprendizaje. Aula de Innovación Educativa, 187, 37-41.

España, E., Rueda, J.A. y Blanco, Á. (2013). Juegos de rol sobre el calentamiento global. Actividades de enseñanza realizadas por estudiantes de ciencias del Máster en Profesorado de Secundaria. Revista Eureka sobre Enseñanza y Divulgación de las Ciencias, 10, 763-779. Recuperado de 21 de octubre de 2014 de http://www.redalyc.org/pdf/920/92028937018.pdf

Exley, K. y Dennis, R. (2007). Enseñanza en pequeños grupos en educación superior. Madrid: Narcea.

Gaete-Quezada, R.A. (2011). El juego de roles como estrategia de evaluación de aprendizajes universitarios. Educación y Educadores, 14(2), 289-307. Recuperado el 12 de noviembre de 2014 de http://educacionyeducadores.unisabana.edu.co/index.php/eye/article/download/1923/ 2489

García, L. y Occelli, M. (2012). Argumentar en la formación profesional continua: un curso de capacitación para docentes de Biología y Química. RILL Nueva época, Prácticas discursivas a través de las disciplinas, 17(1/2). 
Gavaldà, A., Conde, C., Girondo, L., Macaya, A. y Viscarro, I. (2008). La mejora de la capacidad argumentativa en alumnos de Magisterio. En R.M. Ávila, M. Alcázar y M.C. Díez (Eds.), Didáctica de las ciencias sociales, currículo escolar y formación del profesorado. La didáctica de las ciencias sociales en los nuevos planes de estudio (pp. 205-216). Jaén: La casa del libro.

Gunawardena, C., Plass, J. y Salisbury, M. (2001). Do we really need an online discussion group? En D. Murphy, R. Walker y G. Webb (Eds.), Online Learning and Teaching with Technology. Case Studies, Experience and Practice (pp. 36-43). Londres: Kogan Page Ltd.

Hou, H.-T. (2012). Analyzing the Learning Process of an Online Role-Playing Discussion Activity. Educational Technology \& Society, 15(1), 211-222. Recuperado el 2 de noviembre de 2014 de http://www.ifets.info/journals/15_1/19.pdf

Jordán, J. y Peñas, J.A. (2012). El "role-playing" en los estudios de política internacional y resolución de conflictos. REJIE: Revista Jurídica de Investigación e Innovación Educativa, 5, 207-217. Recuperado el 7 de noviembre de 2014 de http://www.eumed.net/rev/rejie/05/jepr.pdf

Krain, M. y Lantis, J. (2006). Building knowledge? Evaluating the effectiveness of the global problems summit simulation. International Studies Perspective Journal, 7(4), 395-407.

López Aymes, G. (2012). Pensamiento crítico en el aula. Docencia e Investigación, 22, 41-60. Recuperado el 10 de noviembre de 2014 de

http://educacion.to.uclm.es/pdf/revistaDI/3_22_2012.pdf

Martín, X. (1992). El role-playing, una técnica para facilitar la empatía y la perspectiva social. Comunicación, lenguaje y educación, 15, 63-67. Recuperado el 8 de noviembre de 2014 de http://dialnet.unirioja.es/descarga/articulo/126264.pdf

Monsalve, M.E. (2012, septiembre). Habilidades argumentativas en la producción de textos con características discursivas multimodales. Comunicación presentada en el Congreso Iberoamericano de las Lenguas en la Educación y en la Cultura / IV Congreso Leer.es, Salamanca, España. Recuperado el 5 de noviembre de 2014 de http://www.oei.es/congresolenguas/comunicacionesPDF/Monsalve_MariaElicenia.pd $\mathrm{f}$

Martínez Riera, J.R. (2009). Influencia del Role-Playing en el proceso de enseñanzaaprendizaje de Enfermería (tesis doctoral inédita). Universidad de Alicante, España. Recuperado el 21 de octubre de 2014 de http://rua.ua.es/dspace/bitstream/10045/14152/1/Tesis_riera.pdf

Morales, P. y Landa, V. (2004). Aprendizaje basado en problemas. Theoria, 13, 145-157. Recuperado el 14 de octubre de 2014 de http://www.unalmed.edu.co/ compedminas/recursos/art\%EDculoABP.pdf

Novelino, J. (2010). Webgincana: un uso estructurado de la web para la educación. En 
C. Barba y S. Capella (Coords.), Ordenadores en las aulas. La clave es la metodología (pp. 163-190). Barcelona: Graó.

Olmos, A. y Martínez, R. (2014). Plataformas virtuales e innovación docente universitaria: Affordance de una Guía de Trabajo Autónomo en Antropología para trabajar competencias de intervención en contextos de diversidad. RED - Revista de Educación a Distancia, 42, 1-15. Recuperado el 14 de octubre de 2014 de http://www.um.es/ead/red/42/olmos_martinez.pdf

Paul, R. y Elder, L. (2006). The Miniature Guide to Critical Thinking, Concepts and Tools. Sonoma State University: Foundation for Critical Thinking.

Pérez Gutiérrez, M. (2004). La dramatización como recurso clave en el proceso de enseñanza y adquisición de las lenguas. Glosas didácticas, 12, 70-80. Recuperado el 12 de noviembre de 2014 de http://www.um.es/glosasdidacticas/doces/GD12/04mapegu.pdf

Pérez-Pérez, I., del Moral, G. y Povedano, M.A. (2014). La mediación en Educación Social a través de una metodología participativa: construcción colaborativa del conocimiento. Revista de Formación e Innovación Educativa Universitaria, 7(3), 117-129. Recuperado el 12 de noviembre de 2014 de http://webs.uvigo.es/refiedu/Refiedu/Vol7_3/7_3_1.pdf

Prawda, G. (2011). El uso del diálogo socrático en el ámbito educativo. P@kenredes, Revista Digital del Centro de Profesores de Alcalá de Guadaíra, 1(9), 1-7.

Recuperado el 5 de octubre de 2014 de http://pakenredes.cepalcala.org/upload/file_aj15_06_11_6_17_06.pdf

Rivas Navarro, M. (2008). Procesos cognitivos y aprendizaje significativo. Madrid: Subdirección General de Inspección Educativa.

Rodríguez, J., Govea, J.E. y Serra, C. (2013). Las vivencias del contexto en los juegos de roles. Revista Mendive, 45. Recuperado el 6 de octubre de 2014 de http://www.revistamendive.rimed.cu/nfuentes/num45/pdf/Art_14_Justina.pdf

Tedesco, J.C. (2011). Los desafíos de la educación básica en el Siglo XXI. Revista Iberoamericana de Educación, 55, 31-47. Recuperado el 2 de octubre de 2014 de http://www.rieoei.org/rie55a01.pdf

Zohar, A. (2006). El pensamiento de orden superior en las clases de ciencias: objetivos, medios y resultados de investigación. Enseñanza de las Ciencias, 24(2), 157-172. Recuperado el 11 de noviembre de 2014 de http://ddd.uab.cat/pub/edlc/02124521v24n2/02124521v24n2p157.pdf 